\title{
What's new for injection drug users with HIV infection?
}

\section{S M Clarke, F M Mulcahy}

\section{Difficulties of providing long term medications to this unique group of patients}

\begin{abstract}
njection drug use represents the risk factor for human immunodeficiency virus (HIV) infection for the minority of worldwide HIV disease. ${ }^{1}$ In contrast, injection drug use in developed countries accounts for between $30 \%$ and $60 \%$ of the HIV infected population. ${ }^{1}$ Much of the published HIV literature focuses on large randomised clinical trials of highly active antiretroviral therapy (HAART) regimens, potential adverse reactions from HAART, and the basic science of HIV infection. There is generally a paucity of research focusing exclusively on injecting drug users (IDUs). A literature review was undertaken to review published data from 1999 to 2001, focusing on articles that directly relate to IDUs and HIV infection. Most of the derived data relate to the following areas:
\end{abstract}

(1) Increasing epidemics of HIV infection in IDUs in developing countries

(2) Improving access to care for IDUs

(3) Co-infection with hepatitis C virus

(4) Pharmacokinetic interactions between antiretrovirals and methadone.

\section{INCREASING EPIDEMICS OF HIV INFECTION IN IDUs IN DEVELOPING COUNTRIES}

Until recently, the concept of IDU was essentially a Western world phenomenon, concentrated in regions of the United States and Western Europe where HIV infection is mainly subtype B disease. In developing countries and countries in political transition where HIV infection was previously vastly under-reported, and IDU was not a significant problem, it is only now that real epidemics of HIV infection among IDUs are occurring and being accurately reported. ${ }^{1}$ Molecular methods and phylogenetic analysis are helping to trace and follow the epidemics.

In many parts of Asia, the HIV epidemic has spread extensively since the 1980s, with multiple, genetically divergent subtypes, but with a predominance of subtype A. ${ }^{2}$ In China, a similar epidemic has been reported with subtype C predominating. Lai et al describe data from Guang Xi province in China, where there has been a threefold increase in the incidence of HIV infection in IDUs between 1998 and 1999. ${ }^{3}$ The authors directly relate this to the rapid adoption of injecting drug use among younger and male heroin users in the area. They also identified needle sharing activity as particularly common among this group of IDUs. There is also evidence of an HIV epidemic affecting IDUs in Ho Chi Minh City, Vietnam. ${ }^{4}$ In a series of three cross sectional studies of IDUs in Ho Chi Minh City between 1995 and 1998, the prevalence rate of HIV infection was $44 \%$ and $38.5 \%$ for IDUs "on the street" and "in the rehabilitation centre" respectively. The authors also identified significant rates of needle sharing and use of "shooting galleries" within this population. An explosive epidemic of HIV-1 has also been documented among IDUs in the former Soviet Union republics, with the Russian Federation currently experiencing the fastest growing HIV epidemic in the world. The two largest epidemics have occurred in the Moscow and Irkutsk regions in the Russian Federation, where $44 \%$ of all HIV infections in Russia have been identified. ${ }^{5}$ Here, subtype A predominates. Similar data from the republic of Maldova describe a predominance of subtype A disease, while the Romanian epidemic among IDUs is mainly subtype F. ${ }^{6}$ There is also evidence for rapid spread of HIV infection in IDUs in Belarus, Kazakhstan, Ukraine, India, and Malaysia. ${ }^{7-9}$ Factors responsible for the rapid spread in epidemic proportions among IDUs in these countries, centres around the concept of the establishment of a "risk environment." Key factors within this environment include rapid diffusion within the cohort, population migration and mixing, economic transition and decline, increasing unemployment and impoverishment, declines in public health revenue, and political, ideological, and cultural transition. This highlights the urgent need for interventions targeting these areas. Without such focused intervention, the prevalence of HIV infection among IDUs will reach levels comparable to that of the heterosexual risk groups of African nations. epidemic has progressed as least one decade ahead of such countries in transition, the question must be asked as to whether the epidemic of HIV infection in IDUs is now under control.

After the original epidemic of HIV infection in IDUs was identified, many countries acted promptly with huge resources focused towards drug treatment services, methadone maintenance programmes, and needle exchange services. Initial results of the benefits from these efforts have yielded conflicting results.

In Amsterdam, after the introduction of intensive prevention and treatment measures, there was a significant reduction in risk behaviour among IDUs between 1986 and 1991. ${ }^{10}$ However, while the rate of behavioural change significantly reduced between 1991 and 1993, there has been no substantial change in risk behaviour or HIV seroprevalence since that time. Vancouver introduced the largest needle exchange programme (NEP) and street nurse programme in North America in 1988. ${ }^{11}$ While the seroprevalence of HIV infection remained stable initially, there has been a rapid increase in seroprevalence since 1994. Strathdee et al have also demonstrated that $40 \%$ of both HIV seropositive and seronegative IDUs persist with needle sharing and lending. ${ }^{12}$ Data from Dublin also demonstrate a recent increase in the incidence of HIV infection in IDUs; however, the actual increase is difficult to estimate. ${ }^{13}$

Other centres have had more success with their programmes for HIV prevention in IDUs. Des Jarlais et al have described five cities (Glasgow, Lund, Sydney, Tacoma, Toronto) able to maintain a low seroprevalence of HIV, all centres having common prevention strategies-implementation of prevention activities when seroprevalence of HIV was still low, provision of sterile injecting equipment, and community outreach programmes to IDUs. ${ }^{14}$ The same authors have outlined data from New York City, where the seroprevalence of HIV infection remains stable with a rate of 56\% between 1984 and $1992 .{ }^{15}$ They also described data that those patients participating in NEPs are three times less likely to be HIV positive than those not participating. More recent data from the same group describe a decline in the epidemic among IDUs in New York City. The authors describe a declining phase (current incidence rate of one per 100 person years) characterised by low incidence and declining prevalence, and the authors suggest that very large seroprevalence HIV epidemics may be reversed. ${ }^{16}$ In the same period they also demonstrate a significant reduction in needle sharing activity from $46 \%$ in 1990 to $24 \%$ in 1997 . Some other countries 
have also reported stabilisation of their HIV incidence data in IDUs, with Italy describing stable incidence rates between 1993 and 1999. ${ }^{17}$ Cities with most success in averting or reversing HIV epidemics among IDUs appear to have introduced intervention methods that emphasise rapid reorientation towards "user friendly" and "low threshold," community based approaches, in conjunction with public policies and funding supportive of such interventions.

\section{IMPROVING ACCESS TO CARE FOR IDUs}

The current standard of care for the treatment of HIV infection involves combination antiretroviral therapy with nucleoside reverse transcriptase inhibitors (NRTIs), nucleotide reverse transcriptase inhibitors, non-nucleoside reverse transcriptase inhibitors (NNRTIs), and protease inhibitors. ${ }^{18}$ Published data show that morbidity and mortality related to HIV infection have significantly reduced since the introduction of such therapy. ${ }^{1 .}$ Unfortunately, many of the large clinical trials assessing the efficacy of such therapy either exclude IDUs from the study population, or have only included IDUs from developed countries with presumed subtype B disease. It is therefore not realistic to equate the results of such trials with potential viral efficacy outcomes in all IDUs

Previous studies have demonstrated that IDUs are less likely than other risk groups to receive antiretroviral therapy (ART), increasing to threefold less likely if they are not enrolled in a drug treatment programme. ${ }^{20}$ Both French and Irish studies have confirmed these findings. ${ }^{21}{ }^{22}$ Strathdee et al describe only $40 \%$ of their ART eligible IDUs to be receiving $A R T$, and most of these were only receiving dual therapy, despite the widespread availability of ART in their unit. ${ }^{20}$ That study was reflective of the period 1996-7. More recently the same group in Vancouver demonstrated an increase in uptake of ART by IDUs, with $60 \%$ of IDUs eligible for ART receiving it, but with still $30 \%$ of treatment eligible IDUs not receiving therapy. ${ }^{23}$ Many factors have been associated with poor acceptance of ART, including continued injecting drug use and non-enrolment in a drug treatment clinic

Owing to the absence of large published randomised controlled clinical trials of IDUs and ART, we are left with small cohort studies of the efficacy of ART in this patient population. From the data that are available, there have been conflicting results. Palepu et al have provided some recent data on enhanced uptake of ART among IDUs in Baltimore between 1996 and 1998. ${ }^{24}$ Not only do they demonstrate increased uptake of
HAART by IDUs (pre-1997 40\%, post$199789.2 \%$ ), but they also demonstrate an improved outcome to therapy. Data from this group also suggest that IDU, as a risk factor, was not significantly associated with the time to achieving viral suppression compared with non IDUs. In contrast, Poundstone et al have also recently published results on differences in disease progression in the era of HAART. ${ }^{25}$ They found that for IDUs, disease free survival increased from 16\% to $34 \%$ post-HAART, compared with non-IDUs improving from $65 \%$ to $135 \%$. Therefore, while disease free survival is increased in IDUs, there is significant disparity in the response to therapy for the different risk groups.

Individualising HAART by using novel methods of drug dispensing, utilising once daily dosing when appropriate, increasing accessibility to drug treatment programmes, and an awareness of interactions between methadone and HAART may improve the overall outcome for IDUs

Evidently the delivery of ART to IDUs is fraught with difficulties, as a result of which methods of optimising access to care and adherence with therapy have been explored. This not only includes novel mechanisms of delivering care, but also through enhanced knowledge of the pharmacokinetic issues that surround methadone maintenance therapy and ART. The resolution of the latter can only provide enhanced patient care and both initial and extended acceptance of ART. While there are numerous antiretroviral agents available for use in combination antiretroviral regimens, the optimal regimen remains to be defined. As for any chronic medical condition, drugs must be chosen that will have minimal immediate and long term adverse events, and that have minimal impact on the patients' quality of life. A once daily preparation is therefore preferable, with ease of use, and a degree of forgiveness from drugs with long half lives. Of the currently available antiretroviral drugs, only four are licensed as once daily dosing-ddI, NVP, EFV, and tenofovir, while favourable pharmacokinetic data suggest that d4T, 3TC and, possibly lopinavir/ritonavir and other boosted protease inhibitor combinations may also be appropriate for once daily dosing. Such therapeutic options are prime candidates for programmes of directly observed therapy (DOT).

St James's Hospital in Dublin first described the concept of delivering HAART to IDUs at methadone maintenance clinics. Clarke et al describe a cohort of 39 patients receiving HAART as DOT through their drug treatment clinic. ${ }^{26}$ At 48 weeks of follow up 58\% of a mixed cohort of ART naive and experienced patients had achieved maximal viral suppression $(<50$ copies $/ \mathrm{ml})$. Stenzel et al have also studied a similar cohort of 37 patients in Vancouver who received ART as DOT over a 12 month period. ${ }^{27}$ DOT was not, however, provided at their methadone maintenance clinic, but provided by an outreach worker in the patients' home environment. Fifty six per cent of their original cohort were still receiving treatment at 12 months, with a mean reduction in viral load of 1.28 copies/ml. Both the Vancouver and Dublin studies depend on patients taking their evening medications by self administration. To eliminate the nocturnal element of patient self medication, Fischl et al have studied the efficacy of DOT in a correctional facility where DOT is provided for all doses. ${ }^{28}$ Initial reports from DOT programmes of antiretroviral therapy in the Department of Corrections in the United States, have shown excellent outcomes. Fischl compares the efficacy of combination antiretroviral therapy in two groups of antiretroviral naive patients: patients receiving ART as DOT in the Department of Corrections (50 patients), and patients self administering therapy (SAT) attending the Clinical Research Unit (50 patients). They demonstrated a statistically significant difference in efficacy outcome between the two cohorts after 80 weeks of follow up, with $95 \%$ of the DOT group compared with $75 \%$ of the SAT group below the limit of detection.

Another study confirms the potential strength of ART when taken with complete compliance. Altice et al looked at the administration of ART within a correctional institute in the Connecticut Department of Corrections. ${ }^{29}$ They found the acceptance of ART (80\%) and adherence $(84 \%)$ to HAART among this group of prisoners $(n=205)$ to be high. Interestingly, they noted no difference in adherence among those receiving DOT $(82 \%)$ or SAT $(85 \%)$. But, while the study has just been reported, it is worth noting that the study was performed in 1996 when $79 \%$ of the patients were receiving only monotherapy or dual nucleoside reverse transcriptase inhibitor therapy.

\section{CO-INFECTION WITH HEPATITIS C VIRUS}

The rate of hepatitis $\mathrm{C}$ virus (HCV) co-infection in HIV infected IDUs has been reported as between $60 \%$ and $90 \% .^{30}$ Since the introduction of HAART, treatment of HCV is now seen as being a priority, with newer therapeutic options available for HCV. Essentially, the real concern for IDUs is that the reduced morbidity and mortality associated with HAART as graphically illustrated by 
Palella et al, ${ }^{19}$ will be inversely related to morbidity and mortality from concurrent HCV infection, and not protease inhibitor (PI) therapy (fig l).

The most promising approach to the treatment of HCV has been the introduction of combination therapy with interferon alfa-2b (IFN) and ribavarin. While this therapy has proved efficacious in the HIV negative population, the efficacy of such therapy in HIV co-infected patients is as yet unclear. ${ }^{31}$ Landau et al describe the long term efficacy of combination therapy with IFN and ribavarin in patients with severe chronic hepatitis and HIV infection. ${ }^{32}$ Those with genotype 3a disease had an improved response to therapy with end of treatment response and sustained viral suppression $(0.002$, 0.003 ), but $29 \%$ of patients discontinued treatment early due to significant adverse drug reactions. The overall response rate seen in this cohort was $20 \%$. Nasti et al have shown combination HCV therapy to be well tolerated, with $31 \%$ of patients achieving sustained viral suppression. ${ }^{33}$ Initial problems associated with therapy for HCV, encountered especially with IDUs, include depressive or psychiatric symptoms and issues around self administering subcutaneous injection. Many of these initial problems may be reduced by the introduction of the pegylated form of interferon with once weekly dosing. Studies differ on the efficacy of pegylated interferon with ribavarin treatment in HIV negative patients, but response rates as high as $88 \%$ have been seen with genotype 2 and 3 , as opposed to response rates of $48 \%$ in patients with genotype 1 disease. ${ }^{34}$ Other issues yet to be resolved include an in vitro demonstrated interaction between ribavarin and the NRTIs. ${ }^{35}$ Patient studies are currently under way to determine the pharmacokinetic and clinical significance of this interaction.

Des Jarlais recently produced an article entitled "Hepatitis C among drug users: Déjà vu all over again," suggesting that the delayed response to the introduction of widespread IDU prevention measures throughout the United States (and indeed Europe), will result ultimately in further rapid spread of the infection and limit treatment options for patients. ${ }^{36} \mathrm{He}$ challenges policy makers

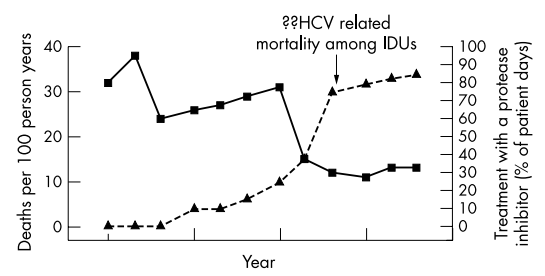

Figure 1 A hypothetical reciprocal relation between the increased use of antiretroviral drugs, and hepatitis $C$ related morbidity and mortality. (Adapted from Palella et a ${ }^{19}$.) to learn from mistakes that have been made, and are still being made during the HIV/AIDS pandemic.

On a more positive note, in a study from Germany, approximately 30\% of IDUs were identified as co-infected with the flavivirus GB virus C (also known as hepatitis G-V), a virus not yet known to cause disease. Fortunately, it was determined that co-infection with $\mathrm{HGV}$ is associated with a reduced mortality rate from HIV infection, and a slower progression to AIDS. This survival benefit remained present even in the years postHAART. $^{37}$ Tillmann et al also demonstrated that $\mathrm{HGV}$ viraemia was a more significant predictor of improved HIV disease outcome compared to those patients with evidence of previous HGV infection but no active viraemia.

\section{PHARMACOKINETIC INTERACTIONS BETWEEN ANTIRETROVIRALS AND METHADONE}

One of the primary concerns for both physicians prescribing HAART to IDUs, and for the IDUs themselves, is whether antiretrovirals interact with methadone. Initial experience, both international medical and "on the street," was that antiretroviral therapy would reduce the effect of methadone, with the consequent emergence of uncomfortable symptoms of opioid withdrawal. The clinical prescribing of methadone is not an exact science, with a poorly defined dose-response curve. Studying the interactions between other drugs and methadone has therefore proved to be difficult. The pharmacokinetic interaction between methadone and the NRTIs has been described; however, studies are limited by the intracellular phosphorylation of the NRTIs, with interpretation of results often difficult. ${ }^{38}$ Methadone is primarily metabolised by CYP 3A4, and the NNRTIs and PIs are potential substrates for this pathway. ${ }^{39}$ A series of four clinical and pharmacokinetic studies have evaluated the interaction between methadone and four commonly prescribed antiretrovirals-nevirapine, efavirenz, nelfinavir, and lopinavir. ${ }^{40-43}$ Pharmacokinetic data, the appearance of methadone withdrawal symptoms, and the need for additional methadone dosing were documented for all patients.

The results of the pharmacokinetic studies demonstrated a highly significant reduction in methadone AUC (0-24 hours) of $60 \%, 50 \%, 37 \%$, and $36 \%$ after the initiation of efavirenz, nevirapine nelfinavir, lopinavir/ritonavir respectively. Clinical data demonstrated that all patients who received nevirapine or efavirenz, $20 \%$ of patients who received nelfinavir, and none of the patients who received lopinavir/ritonavir complained of symptoms of opioid withdrawal after days $7-10$ of therapy. The mean increase in methadone dose prescribed was 10$20 \%$. The reasons for this discrepancy in pharmacokinetic and clinical data for some patients are unclear, and may be related to plasma protein binding, p-glycoprotein, or differing enantiomer effects. A recent study by Gerber et al of the clinical and pharmacological effects of ritonavir and saquinavir on methadone metabolism has provided new data of the effect of plasma protein binding on methadone. ${ }^{44}$ They demonstrated a significant discordant effect on $\mathrm{R}$ and $\mathrm{S}$ isomers with a $40 \%$ reduction in S-methadone compared to a $32 \%$ reduction in R-methadone. Unlike other studies of methadone metabolism, they also corrected for changes in plasma protein binding associated with ritonavir/ saquinavir therapy and, interestingly, demonstrated the actual reduction in free methadone isomers to be $24.6 \%$ for S-methadone and $19.6 \%$ for R-methadone. Further research is required in this area to specifically clarify the pharmacodynamics of this interaction between antiretrovirals and methadone.

Even by utilising programmes of DOT, and anticipating interactions between antiretrovirals and methadone, we are still failing to meet the requirements of many patients from this risk group, There remains a cohort of patients who fulfil standard criteria to commence HAART, but fail to access the available services to obtain therapy. Patient friendly and patient convenient methods are already used in many branches of medicine to improve both the uptake of available facilities and to enhance patient care. The "one stop shop" approach, as provided by national breast screening campaign, and multidisciplinary diabetic clinics, obviates the need for numerous clinic appointments, and ultimately enhances the care of their target populations. Equally, for IDUs, it is essential that we provide "patient friendly" facilities to further improve their access to care. One of the significant practical problems seen in the Dublin cohort is the lack of proximity between the drug treatment clinics and the HIV clinic. Traditionally, drug treatment clinics are situated off hospital grounds, away from the general medical services. A potential way to improve this would be to develop a unit within the hospital setting, where patients could simultaneously access drug treatment programmes and HIV assessment clinics.

The paradigm of HIV treatment has shifted from simply treating HIV infected patients to optimising patient care by individualising patient care. The real challenge for all of us involved in the care of HIV infected patients is the treatment of those patients marginalised in 
society-that is, IDUs, people of colour, refugees, and asylum seekers. Achieving this demonstrates an ability to truly individualise therapy for a patient with particular needs. Perhaps it is no longer appropriate to define treatment success in terms of non-selected patient cohorts but rather on our ability to individualise therapy to those HIV infected patients from marginalised groups within our society.

Sex Transm Infect 2003;79:80-83

\section{Authors' affiliations}

S Clarke, Department of Infectious Diseases, Boston Medical Center, 1 Boston Medical

Center Place, Dowling 3N, Room 3211 , Boston, MA 02118, USA

F Mulcahy, GUIDE Clinic, St James's Hospital, James's Street, Dublin 8, Ireland

Correspondence to: Susan Clarke;

Susan.Clarke@bmc.org

\section{REFERENCES}

1 UNAIDS/ WHO. AIDS epidemic update.

December 2001.

2 Ling $S$, Graf $M$, Zhang $Y$, et al.

Characterization of a virtually full-length HIV type-1 genome of a prevalent intersubtype (C/ $\left.\mathrm{B}^{\prime}\right)$ recombinant strain in China. J Virol 2000;74:11367-76.

3 Lai S, Liu W, Chen J, Yang J, et al. Changes in HIV-1 incidence in heroin users in Guangxi province, China. J AIDS 2001;26:365-70.

4 Hien NT, Giang LT, Binh PN, et al. Risk factors of HIV infection and needle sharing among injecting drug users in Ho Chi Minh City, Vietnam. J Subst Abuse 2001;13:45-58.

5 Bobkov A, Kazennova E, Khanina T, et al. An HIV type-l subtype strain of low genetic diversity continues to spread among injecting drug users in Russia: study of the new local outbreaks in Moscow and Irkutsk. AIDS Res Hum Retrovir 2001;17:257-61.

6 Pandrea I, Descamps D, Collin G, et al. HIV type-1 genetic diversity and genotypic drug susceptibility in the republic of Moldova. AIDS Res Hum Retrovir 2001;13:1297-304.

7 Saraswathy TS, Ng KP, Sinniah M. HIV type-1 subtypes among Malaysian intravenous drug users. Southeast Asian J Trop Med Public Health 2000;2:283-6.

8 Panda S, Chatteriee A, Bhattacharya SK, et al. Transmission of HIV from injecting drug users to their wives in India. Int J STD AIDS 2000; 7:468-73

9 Rhodes T, Ball A, Stimson GV, et al. HIV infection associated with drug injecting in the newly independent states, eastern Europe: the social and economic context of epidemics. Addiction 1999;94:1323-36.

10 Van Ameijden EJC, Coutinho RA. Maximum impact of HIV prevention measures targeted a injecting drug users. AIDS 1998;12:625-33.
11 Strathdee SA, Patrick DM, Currie SL, et al. Needle exchange is not enough:lessons from the Vancouver injecting drug use study. AIDS 1997; 11:F59-65

12 Clarke S, Keenan E, Bergin C, et al. The changing epidemiology of HIV infection in injecting drug users in Dublin, Ireland. HIV Med 2001;2:236-40.

13 Strathdee SA, van Ameijden EJC, Mesquita $F$, et al. Can HIV epidemics among injecting drug users be prevented? AIDS 1998;12:S71-9.

14 Des Jarlais DC, Hagan H, Friedman SR, et al. Maintaining low HIV seroprevalence in populations of injecting drug users. JAMA 1995;274:1226-31.

15 Des Jarlais DC, Friedman S, Sotheran JL, et al. Continuity and change within an HIV epidemic. Injecting drug users in New York City, 1984 through 1992. JAMA 1994;2:121-7.

16 Des Jarlais D, Marmor M, Friedman $P$, et al. HIV incidence among injection drug users in New York City, 1992-1997: evidence for a declining epidemic. Am J Public Health 2000;90:352-9.

17 Sabbatini A, Carulli B, Villa M, et al. Recen trends in the HIV epidemic among injecting drug users in Northern Italy, 1993-1999. AIDS 2001;16:2181-5.

18 Carpenter CC, Cooper DA, Fischyl MA, et al. Antiretroviral therapy in adults:updated recommendations of the International AIDS Society USA panel. JAMA 2000;283:38190.

19 Palella FJ, Delaney KM, Moorman AC, et al. Declining morbidity and mortality among patients with advanced HIV infection. NEngl J Med 1998:338:853-60.

20 Celentano DD, Vlahov D, Cohn S, et al. Self-reported antiretroviral therapy in injection drug users. JAMA 1998;280:544-6.

21 Carrieri MP, Moatti JP, Vlahov D, et al. Access to antiretroviral treatment among French HIV infected injection drug users: the influence of continued drug use. J Epidemiol Commun Health 1999:53:4-8.

22 Clarke S, Delamere S, McCullough L, et al. Assessing limiting factors to the acceptance of antiretroviral therapy in a large cohort of injecting drug users. HIV Med 2003;4:33-7.

23 Wood E, Tyndall MW, Spittal PM, et al. Unsafe injection practices in a cohort of injection drug users in Vancouver: could safer injecting rooms help? CMA 2001;165:405-10

24 Palepu A, Yip B, Miller C, et al. Factors associated with the response to antiretroviral therapy among HIV-infected patients with and without a history of injection drug use. AIDS 2001;15:423-4.

25 Poundstone KE, Chaisson RE, Moore RD. Differences in HIV disease progression by injection drug use and by sex in the era of HAART. AIDS 2001;15:1115-23.

26 Clarke S, Keenan E, Ryan M, et al. Directly observed antiretroviral therapy for injection drug users with HIV. The AIDS Reader 2002:12:305-7, 312-16.

27 Stenzel MS, McKenzie M, Adelson J, et al. Enhancing Adherence to HAART: Modified directly observed therapy. The AIDS Reader 2001;6:317-28.

28 Fischl M, Rodriguez A, Scerpella E, et al. Impact of directly observed therapy on outcomes in HIV clinical trials. Abstract 71:7th Conference on Retroviruses and Opportunistic Infections, San Francisco, Jan 2000.

29 Altice FL, Mostashari F, Friedland GH. Trust and the acceptance of and adherence to antiretroviral therapy. J AIDS 2001; 28:47-58

30 Patrick D, Tyndall MW, Cornelisse PGA, ef al. Incidence of hepatitis $C$ virus among drug users during an outbreak of HIV infection. CMAJ 2001;165:889-95

31 Laver GM, Walker B. Hepatitis C virus infection. N Engl J Med 2001;345:41-52.

32 Landau A, Batisse D, Piketty C, et al. Long-term efficacy of combination therapy with interferon-alpha $2 b$ and ribavarin for severe chronic hepatitis $C$ in HIV-infected patients. AIDS 2001;16:2149-55.

33 Nasti G, Di Gennaro G, Tavio M, et al. chronic hepatitis $C$ in HIV infection: feasibility and sustained efficacy of therapy with interferon alpha-2b and tribavirin. AIDS 2001;15:1783-7.

34 Kewn S, Hoggard PG, Henry-Mowatt JS, et al. Intracellular activation of

$2^{\prime}, 3^{\prime}$-dideoxyinosine and drug interactions in vitro. AIDS Res Hum Retrovir 1999;15:793-802.

35 Des Jarlais D, Schuchat A. Hepatitis C among drug users: déjà vu all over again? Am J Public Health 2001;91:21-2.

36 Tillmann HL, Heiken $\mathrm{H}$, Knapik-Botor $\mathrm{A}$, et al. Effect of coinfection with $G B$ virus $C$ on survival among patients with HIV infection. $N$ Engl J Med 2001;345:715-24.

37 Rainey PM, Friedland G, McCance-Katz EF , et al. Interaction of methadone with didanosine and stavudine. J Acquir Immune Defic Syndr 2000;24:241-8.

38 Dyer KR, Foster DJR, White JM, et al. Steady-state pharmacokinetics and pharmacodynamics in methadone maintenance patients:comparison of those who do and do not experience withdrawal and concentration-effect relationships. Clin Pharmacol Ther 1999;65:685-94.

39 Clarke S, Mulcahy FM, Tijia J, et al. The pharmacokinetics of methadone in HIV-positive patients receiving the non-nucleoside reverse transcriptase inhibitor efavirenz. Br J Clin Pharm 2001;3:213-7.

40 Clarke S, Mulcahy FM, Tjia J, et al. Pharmacokinetic interactions of nevirapine and methadone and guidelines for the use of nevirapine to treat injecting drug users. Clin Infect Dis 2001;33:1595-7.

41 Clarke S, Mulcahy F, Bergin C, et al. The pharmacokinetic interaction between methadone and nelfinavir. Abstract 276, 5th International Congress on Drug Therapy in HIV Infection. Glasgow, Oct 2000.

42 Clarke S, Mulcahy F, Bergin C, et al. Absence of opioid withdrawal symptoms in patients receiving methadone and the protease inhibitor lopinavir/ritonavir. Clin Infect Dis 2002;34: 1 143-5.

43 Gerber JG, Rosenkranz S, Segal Y, et al. Effect of ritonavir/saquinavir on stereoselective pharmacokinetics of methadone: results of AIDS Clinical Trials Group (ACTG 401). J AIDS

2001;27:153-60. 
HIV services

\section{Commissioning HIV services}

\section{W Huxter}

\section{New challenges and new opportunities}

F or those of us who are concerned about how HIV services are commissioned, change is upon us. The NHS Plan, the National Strategy for Sexual Health and HIV, the abolition of the special funding allocation for NHS services, and the devolution of commissioning responsibility to primary care trusts, will all bring HIV services abruptly into the mainstream of the NHS. It is important that clinicians, managers, people with HIV, and all others with an interest in HIV services understand that it is not simply that the rules have changed; rather, we are now playing a different game altogether.

Historically, providers and commissioners of HIV services have had a number of benefits not available to many other parts of the NHS: dedicated money, a relatively high political profile, an articulate and well organised lobby from people with HIV and voluntary organisations, and identified commissioners with personal commitment to HIV and the time to focus on the development of services. Under this protective carapace, many services have flourished, and many people with HIV (though not all) have access to excellentsupport from their treatment centres.

The start of the current financial year on 1 April 2002 brought in at the same time a series of fundamental changes to the arrangements. The NHS no longer receives any money that is formally identified by the Department of Health as required to be spent on HIV, whether prevention or treatment and care. Instead, HIV services have to be funded from the main funding allocation to the NHS, all of which is channelled through primary care trusts (PCTs).

The skewed distribution of HIV across the United Kingdom means that many of the 301 PCTs in England have very few people with HIV living in their area, and HIV is not routinely cited by primary care as a priority. The 28 strategic health authorities have a broader geographical spread, but do not control any revenue funding; their performance management role is concentrated on NHS Plan targets, only one of which relates to HIV ("To implement the National Strategy for Sexual Health and HIV, once issued"). The Action Plan for the National Strategy issued in July 2002 offers exhortation but little active requirement.
The HIV commissioner (perhaps thankfully) is now an endangered species. Increasingly, this role is subsumed within a broader sexual health and/or specialised services role. This has a clear advantage in making sure that people responsible for HIV commissioning are exposed to the realities of priority setting in the wider NHS, but can lead to dilution of expertise and of ready familiarity with the issues faced by HIV services.

Regional specialised commissioning groups (which outside London are no longer co-terminous with the regional structure for the NHS, based in the four directorates of health and social care) are responsible for taking an overview of the commissioning of specialised services, including HIV treatment and care. Regional specialised commissioning groups bring together strategic health authorities and PCTs to agree priorities for specialised services. In London, the regional specialised commissioning group has supported the establishment of a commissioning consortium for HIV services, which has provided a welcome level of stability for providers and commissioners alike, and has minimised the bureaucracy of maintaining open access to care.

It is not simply that the rules have changed; rather, we are now playing a different game altogether

This series of changes sets a number of key challenges for commissioners and providers alike. We need to work intelligently with the new structures to ensure that HIV services meet agreed standards for care and are planned, commissioned, and delivered in a way that reflects the needs of the changing population of people with HIV in England. There are some basic steps that providers can take to equip themselves to play their part, including finding out how commissioning for HIV services is now organised in their area, and therefore who they need to influence. PCTs need to know how much of an issue HIV is for them, in both their provider and their commissioner roles, and how this will increase in the future, and NHS trusts hold much of this information.
For HIV prevention, we must work together to address the overwhelming capacity pressures in GUM services, to maximise opportunities for earlier diagnosis of HIV and appropriate STI management. This means more money, both revenue and capital, and requires commitment by PCTs (including those without a local GUM service) to prioritise these services and to plan collaboratively across a coherent catchment area.

For treatment and care services, we have to seize the opportunities presented by the national strategy, and define and introduce managed networks of care for people with HIV covering primary, secondary and tertiary care. Voluntary sector and local authority services need to be integrated into these arrangements. The networks must be standards driven, and must improve equity of access, so that all patients can access comprehensive high quality care. No HIV centre can on its own deliver all elements of care, nor should it attempt to do so.

In some areas, it may be relatively straightforward to agree who does what within the network, and where it should happen. Experience suggests that more often it may be difficult. In order to take this change forward, there has to be leverage, which can only exist where PCTs and providers are prepared to work together to sign up to a new model of care. Within London, this is being taken forward at sector level (each sector covering one of the five strategic health authorities) within a structure set by the London-wide HIV consortium and a bespoke HIV strategy for the city; this is a model that has much to commend it.

HIV services have an enviable reputation for their quality, and their ability to innovate. Developments in the care of people with HIV, spearheaded by the introduction of combination therapies, have transformed the prognosis for people who are diagnosed in time to benefit. Against this background, responding to organisational changes in the NHS should be a challenge that we can meet with confidence.

Admittedly, there is potential for trouble. Acute trusts and PCTs will need to be won over to developing and maintaining support for HIV services, in an NHS of centrally determined priorities and HIV as a local and growing financial pressure. Without influential champions for HIV services in providers and commissioners, the future could be distinctly gloomy.

However, if we can get our collective response right, the future can be bright. We should be able to secure services that are better planned and coordinated. HIV services should become less isolated 
from mainstream provision, and be prioritised for support based on an objective case of health need, and outcomes secured. This should result in services becoming more sensitive to the needs of their local populations.
Will Huxter was until July 2002 assistant director for specialised services, based at Islington PCT, and was responsible for commissioning HIV services in North Central London. He is writing in a personal capacity.

\section{Sexual health - health of the nation}

\section{W Adler}

\section{A decade later-a further failure}

$T$ he Health of the Nation: a strategy for health in England (HoN), published in 1992, identified HIV/AIDS and sexual health as one of five priority areas with specific objectives and/or targets being set. ${ }^{1}$ The incidence of HIV infection was to be reduced with no targets set, however, with a specific target to reduce the incidence of gonorrhoea among men and women aged 15-64 years by at least $20 \%$ by 1995 (from 61 new cases per 100000 of the population to less than 49 ), and to reduce the rate of conceptions among females under 16 by at least $50 \%$ by the year 2000 (from 9.5 per 1000 among 13-15 year olds to no more than $4.8)$. Five years on from the publication of HoN, it was pointed out that even though the gonorrhoea target had been achieved, most other sexually transmitted infections had increased since publication. $^{2}$ Additionally, pregnancy rates had not decreased. By 2002, a full decade after HoN, little improvement has occurred, and in most instances, if anything, the nation's sexual health has declined.

\section{GONORRHOEA}

By 1997 the target of 49 new cases of gonorrhoea per 100000 had been

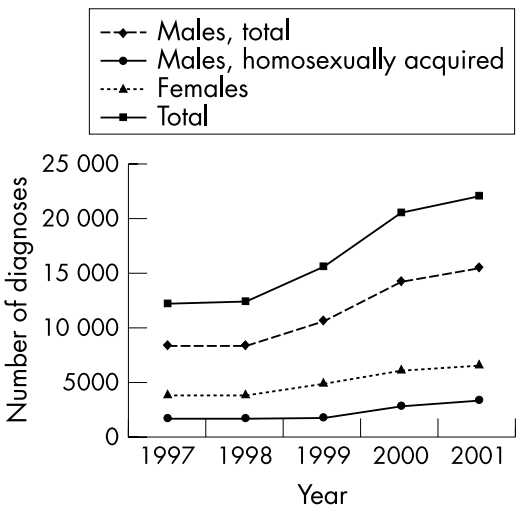

Figure 1 Diagnoses of uncomplicated gonorrhoea by sex, England, 1997-2001. achieved. However, latest figures indicate an increase in both male and female cases of gonorrhoea in England between 1997 and 2001. ${ }^{3}$ There was a 84\% increase in the number of cases in men from 8418 to 15475 , and in females of $67 \%$ from 3981 to 6641 , with an overall increase for both sexes of $78 \%$ (fig 1). This represents an increase in the rate per 100000 for both sexes of $76 \%$ (from 25 in 1997 to 44 in 2001). The incidence of gonorrhoea has increased in homosexual men, as have other STIs, and this is particularly marked in London. In the year $2001,22 \%$ of gonorrhoea diagnoses in males were seen in homosexual men, and in London this was higher at $28 \%$.

\section{CHLAMYDIA}

Chlamydial infection is now the commonest curable bacterial STI seen in England. In the year 2001, 67403 cases of chlamydial infection were diagnosed in departments of genitourinary medicine (GUM), 29154 in males and 38249 in females. This represents an increase of $73 \%$ in the past 5 years (fig 2 ). This condition is most commonly seen in young people; the peak age in men is between 20-24 and between 16-19 in women. Screening surveys carried outside normal STD clinic environments also show high levels in antenatal and gynaecology clinics, general practice, family planning and termination of pregnancy clinics;

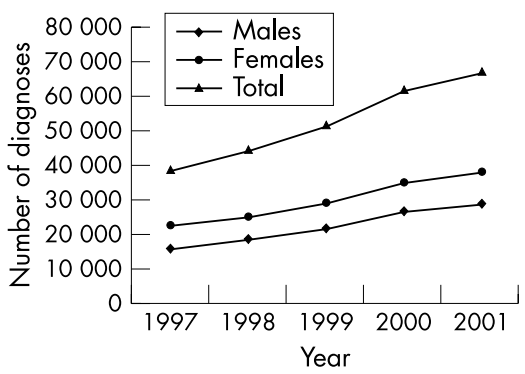

Figure 2 Diagnoses of uncomplicated chlamydia by sex, England, 1997-2001.
Sex Transm Infect 2003;79:84-85

\section{Author's affiliations}

W Huxter, 10 Casino Avenue, London, SE24 9PH, UK; will.huxter@uclh.org

prevalences ranging from 4.5 to $16 \%{ }^{4}$ The two pilot studies of chlamydia screening carried out in the Wirral and Portsmouth reported that $10 \%$ of women under 25 years of age attending health services and being screened were infected with chlamydia.

\section{OTHER STIS}

Anogenital warts are still the commonest viral condition seen in departments of GUM in England with 62222 new cases in 2001. The increase since 1997 is $6 \%$, less than for gonorrhoea and chlamydia. There were 17056 cases of genital herpes in 2001, an increase of $13 \%$ in the past 5 years. Infectious syphilis is now rare in England; however, there has been an increase of $374 \%$ in the past 5 years to a current level of 697 cases diagnosed in 2001, the increase in the past 12 months alone has been $116 \%$. Additionally, a number of outbreaks of syphilis have been reported throughout the country.

\section{HIV/AIDS}

The advent of highly active antiretroviral agents in 1995 has had a profound effect on the progression from HIV to AIDS and subsequent death. The number of new HIV diagnoses has increased dramatically, which means that the prevalent pool of infected individuals is increasing with implications for treatment costs and dangers of onward transmission. The increase in prevalent cases is expected to reach 33930 by 2005 , an average of nearly $10 \%$ per year since 2000 (23017 cases), and a doubling between 1997 and 2005. ${ }^{6}$

The majority of cases of HIV infection and AIDS that have occurred in England have been seen in homosexual men, 54\% and $84 \%$ of the total respectively. Despite this, heterosexual transmission is becoming increasingly important. In 2001 we saw the largest annual total of new diagnoses of HIV (4419) since the start of reporting in $1982,55 \%$ of these were as a result of sex between men and women. Additionally, $79 \%$ of new diagnoses made in heterosexuals were in individuals who had acquired their infection abroad, mainly (71\%) in Africa.

\section{CONCEPTION RATES AND UNWANTED PREGNANCY}

The high rate of teenage conceptions caused considerable concern in the mid 1990s, resulting in the Social Exclusion 


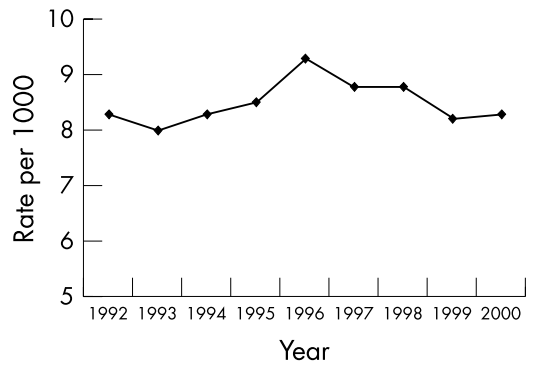

Figure 3 Under 16 years of age conception rates per 1000, England 1992-2000.

Report and the setting up of the Teenage Pregnancy Unit within the Department of Health (DoH) in 1999. ${ }^{7}$ Despite this, the rate in under 16 year old women remains unchanged from 1992, the year of the HoN, and at 8.3 per 1000 in the year 2000 is far short of the target originally set for that year of 4.8 (fig 3 ).

\section{THE CONTEXT/WHAT ELSE IS HAPPENING?}

It is important to put these trends in STIs, HIV, and pregnancy into a context of what changes have occurred in sexual behaviour and service provision within the United Kingdom.

\section{Sexual behaviour}

The recently published National Study of Sexual Behaviour and Lifestyles (NATSAL) has given an indication of the changes that have occurred between 1990 and 2000. ${ }^{89}$ For example, the survey indicates that half of teenagers have sex before the age of 17 years, and that the age of first intercourse has decreased over the 10 years. Additionally, the number of lifetime partners has increased, particularly among females, and the number of individuals involved in concurrent sexual relationships has also increased. It is encouraging that condom use has gone up, but probably not enough to offset the increase in sexual partners. A number of other studies indicate increases in high risk sexual behaviour among homosexual men. ${ }^{11}$

\section{Service pressures}

The increases in infections, pregnancies, and high risk sexual behaviour puts considerable demands on the existing services for STIs and HIV, contraception, abortion, and health promotion.

Clinics for STIs/departments of genitourinary medicine (GUM) have seen a substantial rise in attendances. There has been a doubling in attendances in departments of GUM within England in the past 10 years, now reaching 1.1 million per annum (fig 4). The details of the burden and increases in different diseases have been mentioned earlier, with considerable increases over the past 5 years of diagnoses of chlamydia $(73 \%)$, gonorrhoea $(78 \%)$, and syphilis $(374 \%)$. As a consequence, departments of GUM have found it hard to deliver immediate high quality, open access, and self referral services. Immediate access allows for prompt treatment, avoidance of complications, potential onward transmission, and rapid identification and treatment of sexual partners. The Monks Report of 1988 which looked at GUM workload and infrastructure set a target for patients with a new problem to be seen within 48 hours. ${ }^{12}$ All the evidence indicates that patients are waiting for increasingly longer periods before obtaining an appointment. The length of waiting times has increased within the United Kingdom from 5 days for males and 6 days for females in 2001 to 12 and 14 days respectively by 2002 . These national data do not highlight the particular problems seen in large urban centres, where in some instances patients have to wait over a month for the next available appointment. Additionally, recent surveys indicate that considerable improvements are needed to clinic infrastructure, that one third of clinics operate 3 days a week or less, that the

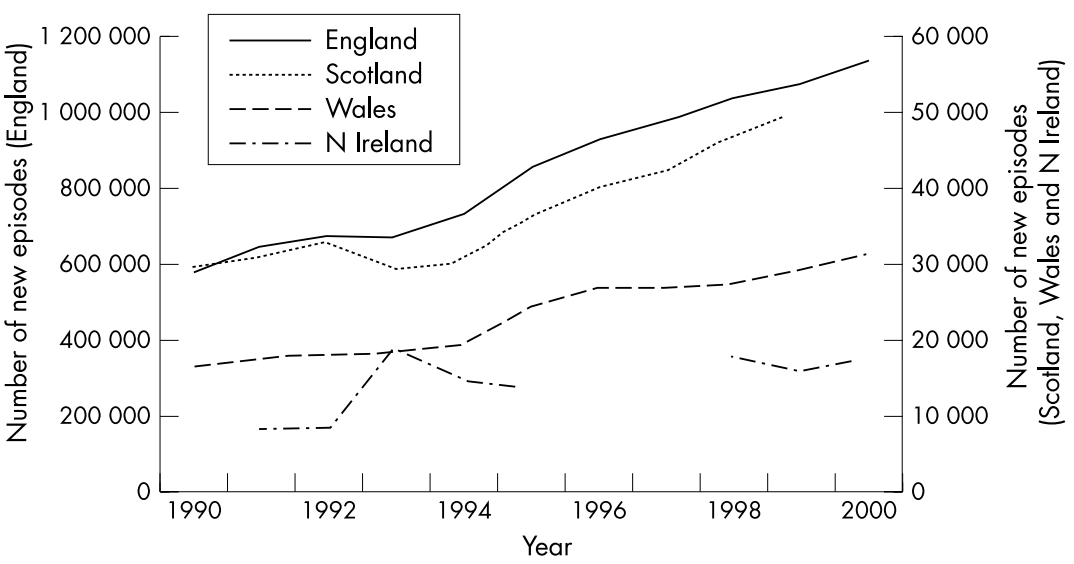

Figure 4 All diagnoses and workload at GUM clinics by country: 1990-2000. (Data are currently unavailable from Scotland for 2000 and from Northern Ireland for 1990, 1996, 1997.)
Summary points

- All sexually transmitted infections have increased in the past 5 years, particularly gonorrhoea $(78 \%)$, chlamydia $(73 \%)$, and syphilis (374\%)

- Prevalent cases of HIV are expected to double between 1997 and 2005

- Teenage pregnancy rates have remained unchanged since 1992

- Changes in sexual behaviour over the past 10 years (decrease in the age of first intercourse, increase in lifetime partners and concurrent relationships, and decrease in safe sex practices among homosexual men) and pressure on GUM services and delays in access will drive the epidemic of STIs

- There is a progressive decline in the nation's sexual health resulting in a public health crisis which will only be resolved by political and financial commitment

Royal College of Physicians' recommendations on consultant expansion in GUM is under target by $70 \%$ resulting in trainees not having consultant posts to apply for (Medical Society for the Study of Venereal Diseases and Association for Genitourinary Medicine. Modernising genitourinary medicine services in England and Wales, personal communication).$^{13-15}$ Clearly all of this is totally unsatisfactory in terms of being able to control sexually transmitted infections and HIV.

Contraception is an important aspect of sexual health in relation to avoiding both unwanted pregnancies and potential sexually transmitted infections. Unfortunately, "the accessibility and range of contraceptive methods available including NHS funded sterilisation vary widely." ${ }^{16}$ In addition, the service is fragmented and of variable quality. Currently, $20 \%$ of NHS contraception prescribing takes place in community clinics, with the remaining $80 \%$ occurring in primary care. Mandatory training is not required for general practitioners providing contraceptive care, but they can be paid on an item for service basis without regard to the quality of service offered. ${ }^{17}$ This hit and miss approach is not ideal for maintaining standards, accessibility of a wide range of contraceptive methods, and tailoring services to users.

As with contraceptive services, there are wide variations in access to abortion services and methods available. The percentage of NHS funded abortions ranges between $46 \%$ and $96 \%$ in different parts of the country and women can wait up to 6 weeks. ${ }^{18}$ These delays militate against 
obtaining an abortion on request and without complications. The current services for terminations fall far short of the Royal College of Obstetricians and Gynaecologists' recommendations. ${ }^{19}$

\section{CONCLUSIONS}

The past decade has seen a continuing and considerable deterioration in the nation's sexual health. All infections have increased alarmingly, teenage pregnancies are yet to decrease, and changes in sexual behaviour regardless of sexual orientation can only continue to drive this situation. It is no exaggeration that we now face a public health crisis in relation to sexual health. The recently published sexual health and HIV strategy for England attempts to outline a plan for better prevention and services. ${ }^{15}$ Only $£ 47.5$ million has been allocated over the next 2 years, not enough to tackle even one aspect of the strategynamely, the roll out of a national screening programme for chlamydia. Concern has been expressed that the allocated resource "is manifestly insufficient.".20 Sexual health is not an NHS or political priority. Until it becomes so we will witness further failure upon further failure.
Sex Transm Infect 2003;79:85-87

Author's affiliations

M W Adler, Department of Sexually Transmitted

Diseases, Royal Free and University College Medical School, The Mortimer Market Centre, off Capper Street, London WC1E 6AU, UK; madler@gum.ucl.ac.uk

\section{REFERENCES}

1 Secretary of State for Health. The health of the nation: a strategy for health in England. London: HMSO, 1992

2 Adler MW. Sexual health-health of the nation failure. BM 1997;314:1743-7.

3 Communicable Disease Surveillance Centre. HIV and STIs: epidemiology 2002. (http.www.phls.co.uk).

4 Department of Health. CMO's expert advisory group on Chlamydia trachomatis. London: $\mathrm{DoH}, 1998$

5 Doherty L, Fenton KA, Jones J, et al. Syphilis: old problem, new strategy. BM 2002;355: 153-6.

6 McHenry A, Evans BG, Sinka K, et al. Numbers of adults with diagnosed HIV infection 1996-2005-adjusted totals and extrapolations for England, Wales and N Ireland. Communicable Disease and Public Health 2002;5:97-100.

7 Social Exclusion Unit. Teenage pregnancy 1999. TSO CM 4342.

8 Johnson AM, Mercer $\mathrm{CH}$, Erens B, et al. Sexual behaviour in Britain: partnerships, practices and HIV risk behaviour. Lancet 2001;358: 1835-42

9 Wellings K, Nanchalal K, Macdowall W, et al. Sexual behaviour in Britain: early heterosexual experience Lancet 2001;358: 1843-50.

10 Dodds JP, Nardone A, Mercey D, et al. Increase in high risk sexual behaviour among homosexual men, London 1996-1998: a cross-sectional questionnaire study. BM 2000;320:1510-1 1

11 Sigma Research. Vital statistics, findings from National Gay Mens Sex Survey 1999. 2000.

12 Department of Health. Report of the working group to examine workloads in GUM clinics. Monks Report. London: DoH, 1998.

13 Diurectic T, Catchpole M, Nicoll A, et al. Genitourinary medicine services in the United Kingdom are failing to meet the current demand. Int J STD AIDS 2001; 12:571-2.

14 Anon. Medical workforce specialty review for genitourinary medicine 2001/2002, England, Wales, Northern Ireland and Scotland. Int J STD AIDS 2002;13:495-8.

15 Medical Workforce Unit, Royal College of Physicians London. Federation of the Royal College of Physicians summary of Information about the consultant workforce in medical specialities in the United Kingdom, 2000. July 2001

16 Department of Health. The national strategy for sexual health and HIV. London: DoH, 2001.

17 Walsh J. Reviewing contraceptive services: research findings and framework. London: Health Education Authority, 1999.

18 British Pregnancy Advisory Service. Personal communication 2001

19 Royal College of Obstetricians and Gynaecologists. National evidence-based guidelines. The care of women requesting induced abortion. 2001

20 Kinghorn G. Sexual health and HIV strategy for England. BM 2001;323:243-4.

\section{Softly, softly does it in promoting sexual health in off street sex workers}

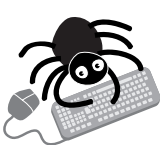

Please visit the Sexually Transmitted Infections website [www.stijournal. com] for link to this full article. aluable lessons are to be learnt in promoting sexual health to women who work "off street" in saunas, massage parlours, and other premises, according to an outreach project doing just that in north west England.

Gaining access is a major hurdle and takes a time and patience. Total honesty about the project and its aims - assessing the needs of this group and offering women health adviceand clear communication are essential. The starting point may be nothing more than delivering condoms to the door. For women who work in their own homes it may entail meetings on neutral territory - in nearby parks or car parks - until rapport and trust are built up.

The caseworker's attitude and demeanour are crucial-a focus on improving the women's sexual health, no underlying attempt to lure them away from the work; an unquestioning acceptance of the work; and respect for the women and willingness to learn from and build on their knowledge.

So far, since the project started in December 1999 the caseworker has achieved unfettered access to four massage parlours and 10 women in their homes. In all, 135 women are contacted regularly and are given contraceptive supplies and advice on sexual health and relevant local services; 21 have been immunised against hepatitis B.

Off street sex workers are a neglected group, yet are estimated to be three times more numerous than their on street counterparts, and they indulge in more high risk behaviours, especially unprotected penetrative sex.

\ Journal of Epidemiology and Community Health 2002;56:903-904. 